\title{
Surface-State Lifetime Measured by Scanning Tunneling Spectroscopy
}

\author{
Jiutao Li* and Wolf-Dieter Schneider \\ Institut de Physique Expérimentale, Université de Lausanne, CH-1015 Lausanne, Switzerland \\ Richard Berndt \\ 2. Physikalisches Institut, RWTH Aachen, D-52056 Aachen, Germany \\ O. R. Bryant and S. Crampin \\ Department of Physics, University of Bath, BA2 7AY, United Kingdom
}

(Received 10 July 1998)

\begin{abstract}
Quasiparticle interactions broaden spectral features at surfaces, which can be measured using the scanning tunneling microscope (STM). We report the first study of lifetime effects on Shockley surfacestate electrons using low-temperature STM spectroscopy. Data taken from $\mathrm{Ag}(111)$ are analyzed using detailed tunneling calculations and a simple model, and are found to correspond to a self-energy of $\Sigma=4.9 \pm 0.6 \mathrm{meV}$. This is considerably below values determined by angle-resolved photoemission, but remains higher than theoretical predictions. [S0031-9007(98)07612-1]
\end{abstract}

PACS numbers: 73.20.At, 61.16.Ch, 72.10.Fk, 73.20.Dx

Noble metal surface states have been the focus of experimental interest for over two decades, from their initial observation by angle-resolved photoemission spectroscopy (ARPES) by Gartland and Slagsvold [1] through to recent studies of defect scattering and lateral confinement to surface nanostructures [2-4]. On the whole, they are very well characterized, with accurate values known for their binding energies, effective masses, and Fermi wave vectors, including the temperature dependence and their response to various changes in surface conditions [5], and these agree well with theoretical predictions. However, the surface-state lifetime $\tau$ remains an elusive quantity, despite prolonged investigation by ARPES [6-9], with current best estimates for inverse lifetimes exceeding theoretical predictions by $\sim 300 \%$ in the case of $\mathrm{Ag}$.

The lifetime is of interest as it determines the mean free path of the surface-state electrons, and hence the effective range of surface-state mediated interactions [10]. Moreover, measurements of $\tau$ have played a fundamental role in honing the analysis of ARPES experiments, one of the key techniques of solid-state spectroscopy, through studies establishing the connection between intrinsic sample properties and recorded peak widths. These are also influenced by finite energy and angular resolution, and importantly surface conditions, and a full understanding is required before ARPES may be confidently used for studying the dynamical properties of many-electron systems, a point cogently highlighted by Smith [11]. The failure to observe the expected lifetimes is despite recent success in achieving a quantitative understanding of the temperature dependence of $\tau$ [7], and an observed correlation between $\tau$ and surface roughness [9], which has enabled an extrapolation to a hypothetical defect-free surface. Indeed this latter work appears to remove the final refuge of most recent attempts to determine $\tau$, which have tended to attribute the remaining discrepancy to imperfect surface conditions, and prompts the question of whether the remaining differences originate in some continuing experimental artifact or point to a true deficiency of theoretical estimates and the underlying assumptions upon which they are made.

With this in mind, we have sought to determine the surface-state lifetime by an alternative technique, using low-temperature tunneling measurements taken from $\operatorname{Ag}(111)$ with the scanning tunneling microscope (STM). The ability of the STM to detect surface topology and to identify minute amounts of contamination, well below the limits of conventional surface analytical techniques, ensures that we study an effectively defect-free surface. Although the surface state is localized at the metal surface, and so might not be expected to carry current, numerous studies have shown otherwise, imaging the surface state spectroscopically [12] as well as mapping out the wave function of the surface state interacting with various surface defects [2-4]. We attribute this to electron-electron and electron-phonon scattering which couple the surface state to extended bulk states, and which give relaxation times much shorter than the time between tunneling events in the $1 \mathrm{nA}$ regime. With lifetime effects included, perturbative theories of the tunneling, which do not distinguish between localized or extended states, become valid [13], and we show below that they provide a consistent account of the STM tunneling data from $\mathrm{Ag}(111)$.

Our experiments are performed in a custom-built ultrahigh vacuum (UHV) STM [14], operating down to temperatures of $5 \mathrm{~K}$ and using electrochemically etched W tips. $\mathrm{A} \operatorname{Ag}(111)$ substrate, cleaned by sputter-anneal cycles in UHV, is scanned to identify a surface region $\left(\geq 3000 \mathrm{~nm}^{2}\right)$ free from defects. Spectroscopy of the differential conductance $d I / d V$ versus the sample voltage $V$ is performed under open feedback loop conditions, adding a voltage modulation (amplitude $V_{m}=1-10 \mathrm{mV}$ at $\omega=230 \mathrm{~Hz}$ ) and using a lock-in amplifier to record 
the signal at $\omega$. In Fig. 1 we show $d I / d V$ spectra measured at $5 \mathrm{~K}$. Figure 1 a shows $d I / d V$ over an extended voltage range, with the sharp rise in the conductance near $-70 \mathrm{meV}$ corresponding to the onset of the contribution from the surface state. This is typical of many taken under a variety of tunneling conditions at $5 \mathrm{~K}$, although the overall slope of the spectrum shows some variation which we attribute to differences in tip structure, tip-sample separation, and drift. This may be seen in Figs. 1b,1c, which show two separately recorded spectra. The width of the onset, which forms the basis of our analysis below, is more consistent from spectrum to spectrum.

To quantify how the lifetime influences spectra such as those in Fig. 1, we calculate the conductance within the framework of the many-body tunneling theory of Zawadowski, Appelbaum, and Brinkman [15], numerically differentiating the current calculated from

$$
\begin{aligned}
I(V)= & \frac{e}{\pi} \int d \mathbf{r}_{S} \int d \mathbf{r}_{S}^{\prime} \int d \omega[f(\omega)-f(\omega+V)] \\
& \times\left[(\bar{\nabla}-\vec{\nabla}) \operatorname{Im} G_{L}\left(\mathbf{r}, \mathbf{r}^{\prime}, \omega+V\right)\right. \\
& \left.\times(\bar{\nabla}-\vec{\nabla}) \operatorname{Im} G_{R}\left(\mathbf{r}^{\prime}, \mathbf{r}, \omega\right)\right] .
\end{aligned}
$$

$G_{R}$ and $G_{L}$ are retarded Green's functions of the tip and sample, $f$ the Fermi function, and the surface integrals are taken over the plane bisecting tip and sample. Following Hörmandinger [13] we approximate the tip by a
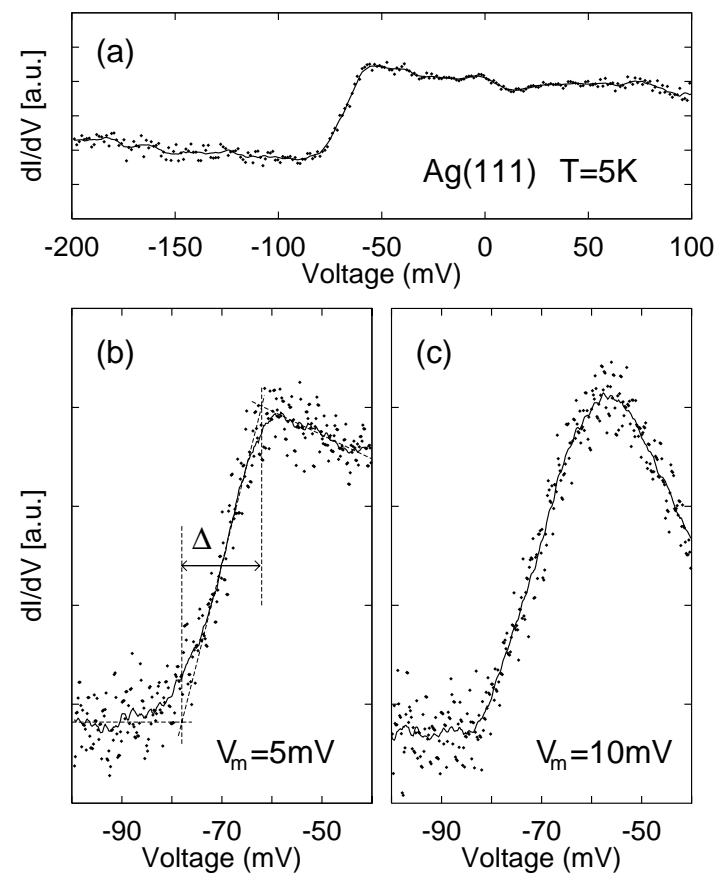

FIG. 1. (a) Experimental $d I / d V$ spectra taken from $\operatorname{Ag}(111)$, showing the steplike onset near $-70 \mathrm{mV}$ due to tunneling into the surface state. (b) and (c) show the onset region in detail from spectra recorded with different tips, showing the variation in slope above the onset. $V_{m}$ is the modulation voltage and in (b) the geometrical width used in the analysis is illustrated. $d I / d V$ doubles just above the onset. single atom, using a $4 \mathrm{eV}$ optical potential to give a realistic spectral density. We calculate the sample Green's function using multiple-scattering techniques treating the crystal within the atomic sphere approximation [16]. Lifetime effects are incorporated via an imaginary self-energy which we treat at an empirical level, using for the selfenergy operator $\Sigma\left(\mathbf{r}, \mathbf{r}^{\prime} ; E\right)$ a local constant term restricted to the sample, with magnitude $\Sigma=\hbar / 2 \tau$. Figure 2 shows conductance spectra evaluated for various values of this self-energy. As $\Sigma$ increases, the onset of the surface-state contribution is seen to broaden, while the relative contribution of the surface state to the conductance above the onset decreases.

As a simple model to show the connection between $\Sigma$ and the width of the onset in $d I / d V$ we write for the tunnel current

$I(V)=C \int_{-\infty}^{\infty} n(E+V)[f(E)-f(E+V)] d \epsilon$,

where $n(E)$ is the surface density of states. This model assumes that we are in the weak coupling regime between the sample and an electronically featureless tip, and that the energy and momentum dependence of the tunneling matrix elements in Eq. (2) can be neglected [17]. Taking for $n(E)$ a constant background $N_{B}$ of bulk states at the surface plus a contribution from the surface state, a two-dimensional electron gas with effective mass $m^{*}$ and binding energy $E_{S}$, and again including lifetime effects via a constant self-energy, then [18] $n(E)=N_{B}+m^{*}[\pi-$ $\left.\phi\left(E-E_{S}\right)\right] / 2 \pi^{2}$ where $\cos \phi(E)=E / \sqrt{E^{2}+\Sigma^{2}}$, and

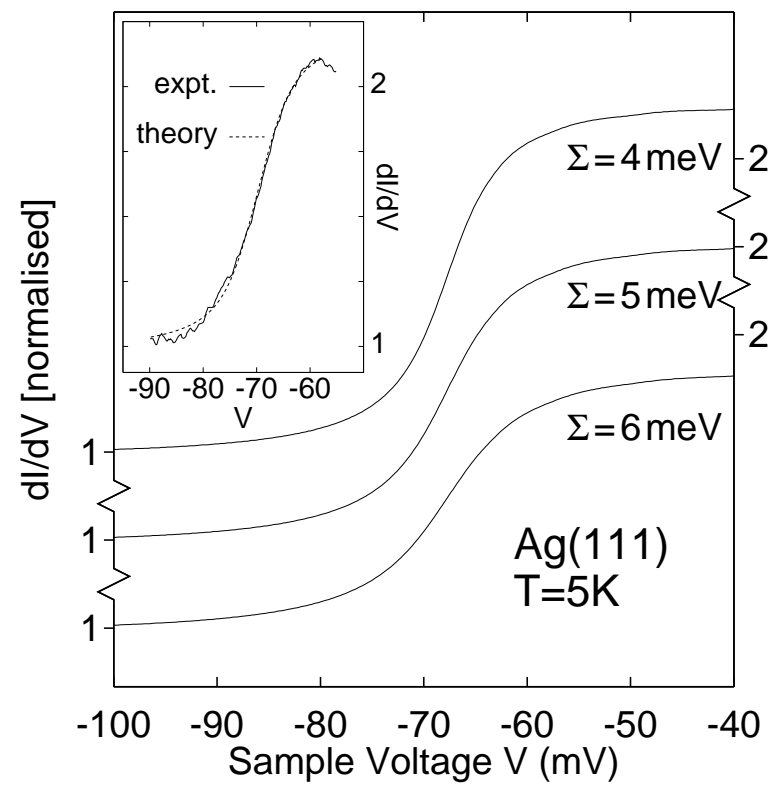

FIG. 2. Calculated $d I / d V$ spectra for various self-energies $\Sigma$. Curves for $\Sigma=4$ and $5 \mathrm{meV}$ are offset for clarity, and each is given relative to the conductance below the surface-state onset. The inset compares the experimental $d I / d V$ spectrum with the theoretical result found with $\Sigma=5 \mathrm{meV}$, convoluted with a $V_{m}=5 \mathrm{meV}$ modulation voltage. 
the differential conductance follows from (2):

$$
\frac{d I}{d V}=C N_{B}+\frac{C m^{*}}{2 \pi^{2}} \int_{-\infty}^{\infty} \frac{\Sigma}{E^{2}+\Sigma^{2}} f\left(E-V+E_{S}\right) d E .
$$

This has the characteristics of Figs. 1 and 2, namely a sharp increase near $E_{S}$, with the width varying with $\Sigma$. To quantify the width, which we denote $\Delta$, we adopt the geometrical definition illustrated in Fig. 1b, extrapolating the slope at the midpoint of the rise to the continuation of the conductance above and below the onset. The variation in the slope found above the surface-state onset in the experimental data, as previously mentioned, prevents a proper "line-shape" analysis, while this definition of the width has the advantage of allowing an analytical treatment. In particular at $5 \mathrm{~K}, T \ll \Sigma$ enabling a Sommerfeld expansion of Eq. (3). This gives

$$
\Delta \simeq \pi \Sigma\left[1+O(T / \Sigma)^{2}\right]
$$

showing a linear relation exists between the geometrical width of the onset and the self-energy [19]. Our detailed calculations using Eq. (2) confirm this result, but with a slope $\simeq 0.9 \pi$, the smaller value reflecting the probability of the finding the electron in the barrier region where $\Sigma\left(\mathbf{r}, \mathbf{r}^{\prime} ; E\right)=0$.

Equation (4) provides the relationship between the measured width of the onset in $d I / d V$ and the selfenergy. However, in our measurements we use the modulation technique to improve the signal-to-noise ratio by eliminating phase-incoherent noise contributions at the expense of an additional source of broadening. This is well understood, with the signal at $\omega$ given by [20]

$$
I_{\omega}=\frac{2}{\pi} \int_{-V_{m}}^{V_{m}} I(V+E) \frac{\sqrt{V_{m}^{2}-E^{2}}}{V_{m}^{2}} d E .
$$

It is possible to apply this convolution to the expression for the model current in Eq. (2) and repeat the above analysis for the width $\Delta$ analytically. This gives $\Delta \simeq$ $\left(\pi V_{m}^{2} / 2\right)\left[\sqrt{\Sigma^{2}+V_{m}^{2}}-\Sigma\right]^{-1}+O(T / \Sigma)^{2}$. For practical purposes $V_{m}$ is in the $1-10 \mathrm{mV}$ range, comparable to $\Sigma$, in which case this result indicates that $V_{m}$ and $\Sigma$ do not combine in a simple manner in determining the overall width. Conversely, given a width $\Delta$ measured using a known modulation voltage $V_{m}$, it is not straightforward to extract the corresponding $\Sigma$. To achieve this we use a plot of $\Sigma$ vs $\Delta$, as shown in Fig. 3. The diagonal lines connecting $\Sigma$ and $\Delta$ are based upon spectra from the detailed tunneling calculations [21], which have been numerically convoluted with various modulation voltages. A measured value of $\Delta$, including uncertainties representing the broadly acceptable range of widths, is related via the appropriate theoretical line to a self-energy. In this way we deduce from three lowtemperature STM spectra values of $\Sigma=6.2 \pm 1.3 \mathrm{meV}$,

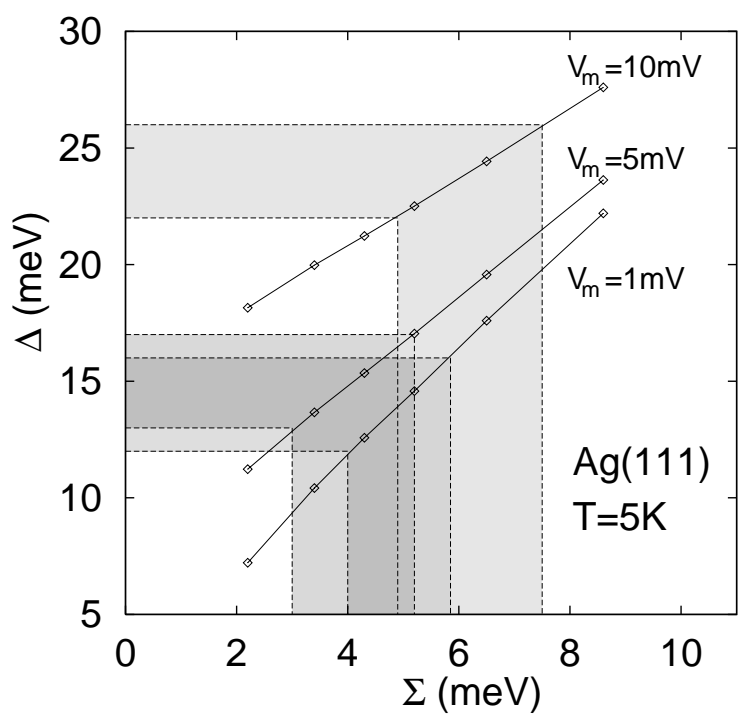

FIG. 3. The solid lines relate the self-energy $\Sigma$ used in the tunneling calculations and the resulting width $\Delta$ of the surfacestate onset, for different modulation voltages $V_{m}$. The shaded regions relate experimentally determined widths, including uncertainties, to corresponding self-energies.

$4.1 \pm 1.1 \mathrm{meV}$, and $4.9 \pm 0.9 \mathrm{meV}$, giving an estimated imaginary self-energy of $\Sigma=4.9 \pm 0.6 \mathrm{meV}$.

Further support for a value around $5 \mathrm{meV}$ comes from the relative contribution of the surface state to the total conductance for voltages above the onset. As may be seen from Fig. 2, for $\Sigma \simeq 5 \mathrm{meV}$ the surface state contributes approximately $50 \%$ of the total signal, which is the contribution observed experimentally. The variation with $\Sigma$ is due to changes in the relative contribution of tunneling into bulk states, with increasing $\Sigma$ resulting in increased spectral weight within the bulk band gap.

Previous attempts to evaluate $\Sigma$ have been by ARPES, where the full width at half maximum of the Lorentzian line shape of the peak associated with the surface state is $\Gamma=2 \Sigma$. The surface state on $\mathrm{Cu}(111)$ has received most attention, with the study by McDougall et al. [7] having the highest resolution. They observed $\Gamma$ varying linearly between $75 \mathrm{meV}$ at $625 \mathrm{~K}$ and $30 \mathrm{meV}$ at $30 \mathrm{~K}$, in quantitative agreement with theoretical predictions of the temperature dependence of the dominant phonon contribution [7]. However, the theory, based on perturbative treatment of the electron-phonon coupling [22] with the Debye model for the phonon spectrum and on Fermi liquid theory for the electron-electron scattering [23] predicts $\Gamma$ to be $14 \mathrm{meV}$ at $T=30 \mathrm{~K}$, and the larger value seen experimentally was attributed to surface roughness [7]. Theilmann et al. [9] have identified an observed correlation between the photoemission linewidth and the width of LEED spots from the same surface, enabling an extrapolation to perfect surface order and yielding $\Gamma=43 \pm 5 \mathrm{meV}$ at room temperature, compared to the theoretical estimate of $\Gamma=30 \mathrm{meV}$. 
For $\operatorname{Ag}(111)$ the most accurate study to date is by Paniago et al. [8], who found $\Gamma=20 \mathrm{meV}$ at $T=56 \mathrm{~K}$. The temperature dependence was not reported, but repeating the calculations performed in Ref. [7] assuming an electron-phonon mass enhancement parameter of 0.13 [22] and Debye energy of $18 \mathrm{meV}$ gives $\Gamma=5 \mathrm{meV}$ at $T=0 \mathrm{~K}$ for states at the surface-state binding energy and only a small increase of the phonon contribution to $\Gamma$ at $T=56 \mathrm{~K}$ of $\sim 1 \mathrm{meV}$. Therefore our value for $\Sigma(=\Gamma / 2)$ of $4.9 \pm 0.6 \mathrm{meV}$ represents a significantly lower experimental estimate of $\Sigma$, but remains above the theoretical value. Whether this remaining difference results from the simplifications used in lifetime calculations or is caused by the measurement via tunneling is an open question.

It is natural to ask whether similar studies might be used to study the energy dependence of $\Sigma$, for example to confirm the expected quadratic dependence with binding energy of the electron-electron scattering contribution $\Sigma_{e e}$ to the self energy [23]. Since on the clean surface it is only the sharp onset at the surface-state binding energy $E_{S}$ which carries the spectroscopic signature of $\Sigma$, in effect yielding $\Sigma\left(E_{S}\right)$, the energy dependence can be identified only through changes in $E_{S}$. As $E_{S}$ rises with temperature [8], it may be possible to perform studies at various temperatures, and hence extract $\Sigma(E)$. However, the larger electron-phonon coupling contribution to the lifetime also increases with temperature, and the thermal broadening increases rapidly, so that it may prove difficult to accurately identify the small contribution from $\Sigma_{e e}$. Another possibility is to shift the position of $E_{S}$ through alloying [24] with some polyvalent solute, but in this case disorder provides an addition contribution to $\Sigma$. This would itself be an interesting subject of study. Finally, we note that the energy dependence of $\Sigma$ might be accessible through the spatial decay of oscillations in $d I / d V$ surrounding surface defects [2].

To summarize, we have reported the first study of lifetime effects on Shockley surface states imaged in the scanning tunneling microscope. We have analyzed data from $\operatorname{Ag}(111)$ using detailed tunneling calculations and a simple model, and identify a value of the electron selfenergy of $\Sigma=4.9 \pm 0.6 \mathrm{meV}$, corresponding to a lifetime $\tau=67 \pm 8 \mathrm{fs}$. This value of the self-energy lies considerably below those determined by angle-resolved photoemission, but remains above theoretical predictions. In view of the high resolution now attainable in scanning tunneling (and photoelectron [25]) spectroscopies, it would clearly be worthwhile to take a fresh look at detailed calculations of surface-state lifetimes and particularly their measurement via tunneling processes.

R. B. thanks J. Braun for useful discussions. J. T. L. and W.D. S. thank the Swiss National Science Foundation for financial assistance, R. B. the SFB 243, and S.C. the EPSRC.
*Present address: Department of Electrical and Computer Engineering, University of Illinois at Urbana-Champaign, 3025 Beckman Institute, Urbana, IL 61801.

[1] P. O. Gartland and B. J. Slagsvold, Phys. Rev. B 12, 4047 (1975).

[2] Y. Hasegawa and Ph. Avouris, Phys. Rev. Lett. 71, 1071 (1993).

[3] M. F. Crommie, C. P. Lutz, and D. M. Eigler, Science 262, 218 (1993).

[4] J. T. Li et al., Phys. Rev. Lett. 80, 3332 (1998).

[5] R. Matzdorf, Surf. Sci. Rep. 30, 153 (1997).

[6] S. D. Kevan, Phys. Rev. Lett. 50, 526 (1983); J. Tersoff and S. D. Kevan, Phys. Rev. B 28, 4267 (1983).

[7] B. A. McDougall, T. Balasubramanian, and E. Jensen, Phys. Rev. B 51, 13891 (1995).

[8] R. Paniago et al., Surf. Sci. 336, 113 (1995).

[9] F. Theilmann et al., Phys. Rev. B 56, 3632 (1997).

[10] N. Memmel and E. Bertel, Phys. Rev. Lett. 75, 485 (1995); E. Bertel, P. Roos, and J. Lehmann, Phys. Rev. B 52, R14384 (1995).

[11] N. V. Smith, Comments Condens. Matter Phys. 15, 263 (1992).

[12] M. P. Everson, R. C. Jaklevic, and W. J. Shen, J. Vac. Sci. Technol. 48, 3662 (1991).

[13] G. Hörmandinger, Phys. Rev. B 49, 13897 (1994).

[14] R. Gaisch et al., Ultramicroscopy 42-44, 1621 (1992).

[15] A. Zawadowski, Phys. Rev. 163, 341 (1967); J. A. Appelbaum and W.F. Brinkman, Phys. Rev. 186, 464 (1969).

[16] S. Crampin, J. Phys. Condens. Matter 5, 4647 (1993).

[17] Valid because in the rate of exponential decay into vacuum, both $e V$ and the energy associated with the momentum of the surface state are much smaller than the tunneling barrier height. This approximation does not affect the surface-state dispersion.

[18] Found from the Green's function for two-dimensional free electrons. See E. N. Economou, Green's Functions for Quantum Physics (Springer-Verlag, Berlin, 1983).

[19] In the $T=0$ limit the model corresponds to identifying $d I / d V$ as $n(V)$. Convoluting a steplike density of states with a Lorentzian of FWHM $2 \Sigma$, and then applying the same definition of the width $\Delta$ provides an alternative route to $\Delta=\pi \Sigma$. The geometrical width of the Lorentzian alone can readily be shown to be $2 \Sigma$, so the additional factor of $\pi / 2$ can be identified as resulting from the distribution of states probed by the STM.

[20] J. Klein et al., Phys. Rev. B 7, 2336 (1973).

[21] These lie below the corresponding analytic result, due to $\Sigma=0$ in the barrier region and the approximations leading to Eq. (5). This leads to slightly larger estimates of $\Sigma$ than predicted with the analytic model.

[22] G. Grimvall, The Electron-Phonon Interaction in Metals (North-Holland, New York, 1981).

[23] D. Pines and P. Nozières, The Theory of Quantum Liquids (Benjamin, New York, 1969).

[24] R. Prasad, A. Y. Serageldin, and A. Bansil, J. Phys. Condens. Matter 3, 801 (1991).

[25] D. Purdie et al., Surf. Sci. 407, L671 (1998). 